\title{
Entangled States in Interferometry
}

\author{
B. Hessmo*, J. SÖDERHOLM AND G. BJÖRK
}

Department of Microelectronics and Information Technology

Royal Institute of Technology, 16440 Kista, Sweden

\begin{abstract}
We show how some entangled quantum states can be used to improve interferometric measurements. We describe why Schrödinger-cat states are very sensitive to relative-phase shifts and why relative-phase states as described by A. Luis and L.L. Sánchez-Soto resolve the interval $[0,2 \pi]$ well. We also describe how the quantum concept of well-defined relative-phase and the classical visibility of an interference pattern are related.
\end{abstract}

PACS numbers: 42.50.Dv

\section{Introduction}

Interferometric measurements is a standard technique where a relative-phase shift between two modes is used to switch between constructive and destructive interference in a mode where a detector is placed. The most simple examples of this are the well-known Michelson and Mach-Zehnder interferometers [1-3]. When a relative-phase shift $\pi$ is introduced between the two interferometer modes (the two arms), the interference between classical waves in the detector mode change from constructive to destructive. We will illustrate some non-classical properties in interferometry when quantum states are used instead of classical waves. Entangled quantum states may enhance the sensitivity of an interferometer beyond what is possible using classical light.

\section{Relative phase}

The unitary operator generating a relative-phase shift between two modes is

$$
\hat{U}_{\mathrm{rps}}(\phi)=\mathrm{e}^{\mathrm{i} \phi \hat{n}_{12}},
$$

where $\hat{n}_{12}=\left(\hat{n}_{1}-\hat{n}_{2}\right) / 2$ is half the population difference between mode 1 and 2 .

*corresponding author; e-mail: hessmo@ele.kth.se 
This operator is easily derived from the Hamiltonians for the two modes, $H_{1}=$ $\hbar \omega_{1} \hat{n}_{1}$ and $H_{2}=\hbar \omega_{2} \hat{n}_{2}$. The time evolution operator is given by

$$
\begin{aligned}
\hat{U}(t) & =\mathrm{e}^{-\mathrm{i}\left(\omega_{1} \hat{n}_{1}+\omega_{2} \hat{n}_{2}\right) t}=\mathrm{e}^{-\mathrm{i}\left(\omega_{1}+\omega_{2}\right)\left(\hat{n}_{1}+\hat{n}_{2}\right) t / 2} \mathrm{e}^{-\mathrm{i}\left(\omega_{1}-\omega_{2}\right)\left(\hat{n}_{1}-\hat{n}_{2}\right) t / 2} \\
& =\mathrm{e}^{-\mathrm{i} \frac{\left(\omega_{1}+\omega_{2}\right) t}{2} \hat{N}} \mathrm{e}^{\mathrm{i} \phi \hat{n}_{12}},
\end{aligned}
$$

with $\hat{N}=\hat{n}_{1}+\hat{n}_{2}$ as the total particle number, and where the relative-phase shift is given by $\phi=\left(\omega_{2}-\omega_{1}\right) t$. The first factor of $\mathrm{Eq}$. (2) represents a global phase change for a two-mode state with fixed particle number. The second factor, $\hat{U}_{\text {rps }}(\phi)$, introduces a relative-phase shift between the two modes depending on how large the particle number difference is. Here the phase-shift is achieved by having different $\omega_{k}$ for the two modes, similar results are obtained for different propagation times for the two modes [4]. The eigenstates to the phase-shift operator are number states

$$
\hat{U}_{\mathrm{rps}}(\phi)|k, l\rangle=\mathrm{e}^{\mathrm{i} \phi(k-l) / 2}|k, l\rangle,
$$

where $|k, l\rangle=|k\rangle_{\text {mode } 1} \otimes|l\rangle_{\text {mode } 2}$ defines the particle numbers for the two modes. We have $\hat{n}_{1}|k, l\rangle=k|k, l\rangle$ and $\hat{n}_{2}|k, l\rangle=l|k, l\rangle$.

We define a two-mode state $|\xi\rangle$ to have a well-defined relative-phase if the operator $\hat{U}_{\text {rps }}(\phi)$ transforms the state $|\xi\rangle$ into an orthogonal state $\left|\xi^{\perp}\right\rangle=\hat{U}_{\text {rps }}(\phi)|\xi\rangle$ for at least one value of $\phi$. See [4] for a more detailed discussion. The obvious mathematical description of this property of $|\xi\rangle$ is

$$
\left\langle\xi\left|\hat{U}_{\mathrm{rps}}(\phi)\right| \xi\right\rangle=0
$$

This definition is operational since it is in principle possible to distinguish the states $|\xi\rangle$ and $\left|\xi^{\perp}\right\rangle$ in terms of the observable

$$
\hat{\Theta}=\theta|\xi\rangle\left\langle\xi\left|+\theta^{\perp}\right| \xi^{\perp}\right\rangle\left\langle\xi^{\perp}\right| \text {. }
$$

In the case when many orthogonal states can be obtained by application of the phase-shift operator we have a general observable distinguishing these states

$$
\hat{\Theta}=\sum_{k} \theta_{k}\left|\xi_{k}\right\rangle\left\langle\xi_{k}\right|
$$

where $\left|\xi_{k}\right\rangle=\hat{U}_{\mathrm{rps}}\left(\phi_{k}\right)\left|\xi_{0}\right\rangle$ with the property $\left\langle\xi_{n} \mid \xi_{m}\right\rangle=\delta_{n m}$.

\subsection{Single photon states}

Let us consider one photon entering an input port of a Mach-Zehnder (MZ) interferometer. The state is described by $\hat{a}_{1}^{\dagger}|0\rangle$. (See Fig. 1 for a definition of the field operators.) After the first beam splitter the photon is in a superposition of being found in the two interferometer arms (modes). Thus the photon state inside the interferometer is

$$
\left|\psi_{1}\right\rangle=\frac{1}{\sqrt{2}}(|1,0\rangle+|0,1\rangle)
$$


where $|1,0\rangle(|0,1\rangle)$ indicates that the photon can be found in the upper (lower) interferometer arm. For simplicity we have ignored phase shifts accumulated during reflections on mirrors and beam splitters. The action of a beam splitter on the field operators is defined by the transformation $\hat{a}_{1} \rightarrow \frac{1}{\sqrt{2}}\left(\hat{b}_{1}+\hat{b}_{2}\right)$ and $\hat{a}_{2} \rightarrow \frac{1}{\sqrt{2}}\left(-\hat{b}_{1}+\hat{b}_{2}\right)$, where $\hat{a}_{k}$ refers to the modes before the first beam splitter and $\hat{b}_{k}$ to the modes after it. Similarly for the second beam splitter. When the phase-shift operator is applied to the state ( 7 ), we have

$$
\hat{U}_{\mathrm{rps}}(\phi)\left|\psi_{1}\right\rangle=\frac{1}{\sqrt{2}}\left(\mathrm{e}^{\mathrm{i} \phi / 2}|1,0\rangle+\mathrm{e}^{-\mathrm{i} \phi / 2}|0,1\rangle\right)
$$

yielding $\left\langle\psi_{1}\left|\hat{U}_{\mathrm{rps}}(\phi)\right| \psi_{1}\right\rangle=\cos (\phi / 2)$. This means that this state has a well-defined relative-phase, since (7) and (8) are orthogonal for $\phi=\pi$. We also note that the two states can be detected by monitoring the output of the MZ-interferometer. When the interferometer is balanced so that $\left|\psi_{1}\right\rangle$, after the second beam splitter, is detected in one of the output modes with certainty, then $\hat{U}_{\mathrm{rps}}(\pi)\left|\psi_{1}\right\rangle$ is detected in the other output mode.

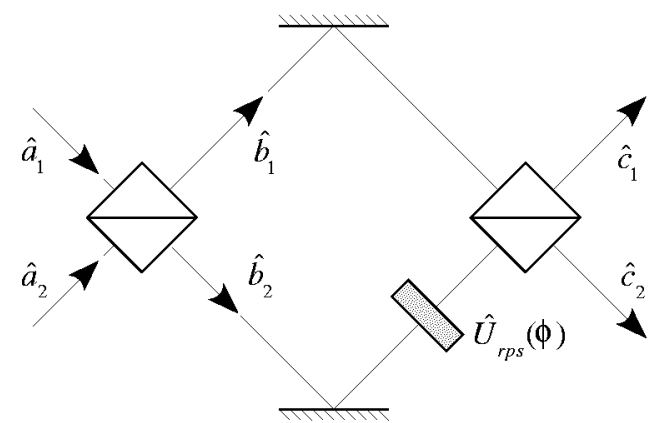

Fig. 1. A Mach-Zehnder interferometer. The field operators for the incoming (outgoing) modes are denoted $\hat{a}_{k}\left(\hat{c}_{k}\right)$. The modes inside the interferometer are described by the operators $\hat{b}_{k}$.

However, if the beam splitter is asymmetric, the state after it becomes

$$
\left|\tilde{\psi}_{1}\right\rangle=\alpha|1,0\rangle+\beta|0,1\rangle
$$

where $\alpha(\beta)$ is the reflection (transmission) amplitude of the beam splitter satisfying the relation $|\alpha|^{2}+|\beta|^{2}=1$. Evaluating formula (4) one has

$$
\left|\left\langle\tilde{\psi}_{1}\left|\hat{U}_{\mathrm{rps}}(\phi)\right| \tilde{\psi}_{1}\right\rangle\right|^{2}=\cos ^{2} \frac{\phi}{2}+\left(|\alpha|^{2}-|\beta|^{2}\right)^{2} \sin ^{2} \frac{\phi}{2}>0
$$

This is greater than zero for $|\alpha| \neq|\beta|$, which means that the state $\left|\tilde{\psi}_{1}\right\rangle$ does not have a well-defined relative-phase. That is, there is no measurement that can distinguish the original and phase-shifted state with certainty. In a classical 
interference experiment this would be seen as a reduced visibility [5] of the interference fringes. Later we will address the relation between visibility and well-defined relative-phase.

\section{2. $N$-photon states}

In the previous section we saw what happened to one-photon states when they are subject to relative-phase shifts inside a Mach-Zehnder interferometer. The generalization to $N$-photon states is straightforward

$$
\begin{aligned}
&|N, 0\rangle=\frac{\left(\hat{a}_{1}^{\dagger}\right)^{N}}{\sqrt{N !}}|0\rangle \\
& \rightarrow\left|\psi_{N}\right\rangle=\frac{\left(\hat{b}_{1}^{\dagger}+\hat{b}_{2}^{\dagger}\right)^{N}}{\sqrt{2^{N}} \sqrt{N !}}|0\rangle=\sum_{n=0}^{N} \sqrt{\frac{N !}{2^{N}(N-n) ! n !}}|n, N-n\rangle .
\end{aligned}
$$

These states are referred to as binomial states [6]. See also [7, 8] for more details on the use of such states. When the phase-shift is applied on a binomial state, one obtains

$$
\hat{U}_{\text {rps }}(\phi)\left|\psi_{N}\right\rangle=\sum_{n=0}^{N} \sqrt{\frac{N !}{2^{N}(N-n) ! n !}} \mathrm{e}^{\mathrm{i}\left(n-\frac{N}{2}\right) \phi}|n, N-n\rangle .
$$

Combining Eqs. (10) and (11) gives

$$
\left\langle\psi_{N}\left|\hat{U}_{\mathrm{rps}}(\phi)\right| \psi_{N}\right\rangle=\cos ^{N}\left(\frac{\phi}{2}\right)
$$

The binomial states have one very special property. When the two modes are combined on a normal 50/50-beam splitter they are the only ones that may exhibit unit visibility ${ }^{\dagger}$. Equation (11) can be rewritten in the form

$$
\begin{gathered}
\hat{U}_{\mathrm{rps}}(\phi)\left|\psi_{N}\right\rangle=\frac{1}{\sqrt{2^{N} N !}} \sum_{n=0}^{N} \frac{N !}{(N-n) ! n !}\left(\mathrm{e}^{\mathrm{i} \phi / 2} \hat{b}_{1}^{\dagger}\right)^{n}\left(\mathrm{e}^{-\mathrm{i} \phi / 2} \hat{b}_{2}^{\dagger}\right)^{N-n}|0\rangle \\
=\frac{1}{\sqrt{N !}}\left(\frac{\mathrm{e}^{\mathrm{i} \phi / 2} \hat{b}_{1}^{\dagger}+\mathrm{e}^{-\mathrm{i} \phi / 2} \hat{b}_{2}^{\dagger}}{\sqrt{2}}\right)^{N}|0\rangle .
\end{gathered}
$$

The second beam splitter transforms this into

$$
\left|\psi_{\text {out }}\right\rangle=\frac{1}{\sqrt{N !}}\left[\sin (\phi / 2) \hat{c}_{1}^{\dagger}+\cos (\phi / 2) \hat{c}_{2}^{\dagger}\right]^{N}|0\rangle= \begin{cases}|N, 0\rangle, & \phi=\pi \\ |0, N\rangle, & \phi=0\end{cases}
$$

Detection of $N$ photons in mode $c_{1}$ and zero photons in $c_{2}$ implies that the relative-phase introduced inside the interferometer was $\pi$. The opposite situation indicates that the phase shift was zero.

\footnotetext{
${ }^{\dagger}$ It is easy to prove that only a binomial state can produce the states $|N, 0\rangle$ and $|0, N\rangle$ after the last beam splitter. Invert the unitary transformation of the beam splitter, apply it to the state $|N, 0\rangle$ or $|0, N\rangle$.
} 


\subsection{Sensitivity to change in relative-phase}

It is of interest to do interferometry with light states that are highly sensitive to a change in the relative-phase. This could ultimately enable us to detect very small changes in the interferometer arms, for instance in a gravitational wave detector [9]. It is possible to increase the sensitivity of the state $\left|\psi_{1}\right\rangle$ in Eq. (7) by adding more particles to each mode. Such Schrödinger-cat energy eigenstates have the form $[10-12]$ :

$$
\left|\Psi_{N}\right\rangle=\frac{1}{\sqrt{2}}(|N, 0\rangle+|0, N\rangle),
$$

and these states require small relative-phase shifts to become orthogonal. We have

$$
\left\langle\Psi_{N}\left|\hat{U}_{\mathrm{rps}}(\phi)\right| \Psi_{N}\right\rangle=\cos \left(\frac{N \phi}{2}\right) .
$$

The sensitivity for a change in relative-phase for Schrödinger-cat states increases with increasing number of particles in the state. A phase shift of $\pi / N$ brings $\left|\Psi_{N}\right\rangle$ to an orthogonal state. However, the phase resolution is not very good, since only two mutually orthogonal states can be obtained applying a relative-phase shift. We also note that it is not possible to distinguish these two states using a regular beam splitter, since the Schrödinger-cat state and the binomial states are different for $N>1$.

Of significant interest for many interferometric applications would be to construct a state such that a number of small relative-phase shifts give several mutually orthogonal states. These could, in principle, be detected with certainty.

This could be achieved by using an equally weighted superposition of the eigenstates of the relative-phase operator, which were defined by Luis and Sánchez-Soto [13]. For $N$ photons such a relative-phase state takes the form

$$
\left|\phi_{r}^{(N)}\right\rangle=\frac{1}{\sqrt{N+1}} \sum_{n=0}^{N} \mathrm{e}^{\mathrm{i} n \phi_{r}^{(N)}}|n, N-n\rangle,
$$

where $\phi_{r}^{(N)}=\phi_{0}^{(N)}+\frac{2 \pi r}{N+1}$, with $r=0,1, \ldots, N$. With $N$ photons these form $N+1$ orthogonal states. Assuming $\phi_{0}^{(N)}=0$ one has for $r=0$

$$
\left|\phi_{0}^{(N)}\right\rangle=\frac{1}{\sqrt{N+1}} \sum_{n=0}^{N}|n, N-n\rangle .
$$

Applying the phase-shift operator on this state one finds

$$
\hat{U}_{\mathrm{rps}}\left(\frac{2 \pi r}{N+1}\right)\left|\phi_{0}^{(N)}\right\rangle=\left|\phi_{r}^{(N)}\right\rangle .
$$

Hence, all the $N+1$ different relative-phase states can be obtained by different relative-phase shifts on one of the states. We see that Eq. (4) becomes 


$$
\left\langle\phi_{r}^{(N)}\left|\hat{U}_{\mathrm{rps}}(\phi)\right| \phi_{r}^{(N)}\right\rangle=\frac{\sin (N+1) \frac{\phi}{2}}{(N+1) \sin \frac{\phi}{2}},
$$

which is zero for $\phi=\frac{2 \pi r}{N+1}$. In Fig. 2 we compare the relative-phase state with the Schrödinger-cat state by plotting the overlap $\left\langle\xi\left|\hat{U}_{\text {rps }}(\phi)\right| \xi\right\rangle$, for $|\xi\rangle=\left|\phi_{r}^{(N)}\right\rangle$ and $|\xi\rangle=\left|\Psi_{N}\right\rangle$ with $N=3$.

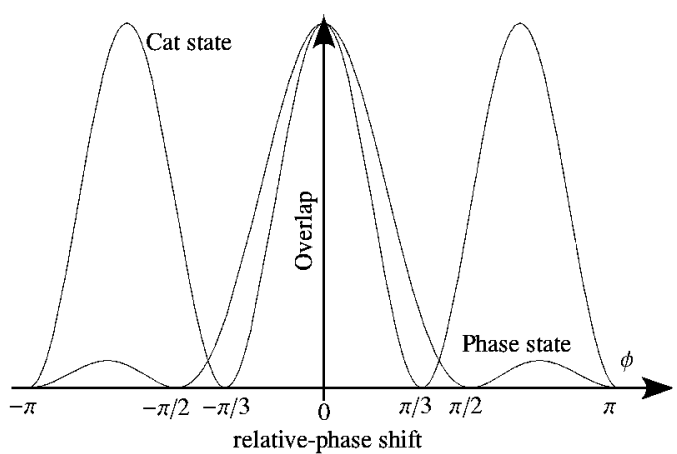

Fig. 2. A plot of $\left\langle\xi\left|\hat{U}_{\text {rps }}(\phi)\right| \xi\right\rangle$ as a function of the relative-phase shift for both the relative-phase state in Eq. (16) and the Schrödinger-cat state in Eq. (15). In both cases the number of photons is $N=3$. Note that the cat state becomes orthogonal more rapidly, and that the phase state does not return to unit overlap as the cat state does (at $\phi= \pm 2 \pi / 3$ ). The three different phase-shifted relative-phase states at $\phi= \pm \pi / 2$ and $\phi= \pm \pi$ are all orthogonal, whereas the points $\phi= \pm \pi / 3$ and $\phi= \pm \pi$ correspond to the same state for the Schrödinger-cat state.

\subsection{Entanglement and relative-phase states}

To produce relative-phase states in the lab is a challenge for high particle numbers. The reason for this is that states of the form

$$
|\psi\rangle=\frac{1}{\sqrt{N+1}} \sum_{n=0}^{N} \mathrm{e}^{\mathrm{i} \theta_{n}}|n, N-n\rangle
$$

are highly entangled. Actually, the states carry only information about correlations between the two modes. This can be seen from the entropy of the reduced density operator for one of the modes. We have $\hat{\rho}_{12}=|\psi\rangle\langle\psi|$. The reduced density operator for mode 1 (2) is formed by the partial trace over mode 2 (1)

$$
\begin{aligned}
& \hat{\rho}_{1}=\operatorname{Tr}_{2}\left[\hat{\rho}_{12}\right]=\frac{1}{N+1} \sum_{n=0}^{N}|n\rangle\langle n|, \\
& \hat{\rho}_{2}=\operatorname{Tr}_{1}\left[\hat{\rho}_{12}\right]=\frac{1}{N+1} \sum_{n=0}^{N}|n\rangle\langle n| .
\end{aligned}
$$


This density operator maximizes the von Neumann entropy $S(\hat{\rho})=-\operatorname{Tr}[\hat{\rho} \log \hat{\rho}]$. For $\hat{\rho}_{1}$ the entropy becomes $\log (N+1)$, meaning that we lack $\log (N+1)$ bits of information about the state if we look at only one of the modes. This is the full capacity of $N+1$ states, so the state carries no information about the individual modes. If both modes are considered we have $S\left(\hat{\rho}_{12}\right)=0$. This means that we have access to $\log (N+1)$ bits of information about the relation between the two modes. This is not surprising, since the relative-phase states express a relation between the two modes, and if we wish to know as much as possible about this relation we should not waste the information capacity of the state to describe properties of the individual modes. We therefore can conclude that the relative-phase states are optimal in resolving relative-phases from this reasoning about the entropy.

To produce entangled photon state with several particles is difficult, the present methods are based on parametric down conversion and may yield four-photon states $[14,15]$. To go beyond that is an experimental challenge. For two photons the relative-phase states have been implemented using down-converted photon pairs [16], a similar technique can also be employed to produce states that are very sensitive to spatial rotations $[17,18]$.

\section{Visibility and well-defined relative phase}

From the previous section we learned that in many cases we can distinguish states with different (well-defined) relative-phases in terms of visibility in an interference experiment. Is this general? First, we note that if the interferometer is a regular Mach-Zehnder interferometer with regular beam splitters, then only the binomial states, and superpositions and mixtures of these, could exhibit unit visibility. However, we may build very general interferometric instruments where the beam splitters are replaced with general lossless unitary transformations acting on the two modes (generalized beam splitters). A general two-mode state can be written

$$
|\xi\rangle=\sum_{N=0}^{\infty} \sum_{n=0}^{N} c_{n, N}|n, N-n\rangle .
$$

We denote the operation of a generalized unitary beam splitter by $\hat{U}_{\text {gbs }}$. The operator must also be lossless so that the number of particle in the two outgoing modes is the same as in the incoming modes. This may act upon the general two-mode state yielding $\hat{U}_{\mathrm{gbs}}|\xi\rangle=\left|\xi^{\prime}\right\rangle$. To reach full visibility we require that it is possible to find a relative-phase shift such that the number of particles in the detector modes reaches zero. This is seen from the definition of visibility

$$
\mathcal{V}=\frac{\langle\hat{n}\rangle_{\max }-\langle\hat{n}\rangle_{\min }}{\langle\hat{n}\rangle_{\max }+\langle\hat{n}\rangle_{\min }}
$$

which becomes unity only if $\langle\hat{n}\rangle_{\min }=0$. This means that the following relations must be satisfied if we wish to separate two states generated by a relative-phase shift using two detectors: 


$$
\begin{aligned}
& \hat{U}_{\mathrm{gbs}} \hat{U}_{\mathrm{rps}}\left(\phi_{1}\right)|\xi\rangle=\mathrm{e}^{\mathrm{i} \zeta}|\Psi\rangle \otimes|0\rangle, \\
& \hat{U}_{\mathrm{gbs}} \hat{U}_{\mathrm{rps}}\left(\phi_{2}\right)|\xi\rangle=\mathrm{e}^{\mathrm{i} \zeta^{\prime}}|0\rangle \otimes|\Phi\rangle,
\end{aligned}
$$

where $\zeta$ and $\zeta^{\prime}$ are possible global phase factors. The scalar product of the left hand sides of Eqs. (21) and (22) becomes

$$
\begin{aligned}
& \left\langle\xi\left|\hat{U}_{\mathrm{rps}}^{\dagger}\left(\phi_{2}\right) \hat{U}_{\mathrm{gbs}}^{\dagger} \hat{U}_{\mathrm{gbs}} \hat{U}_{\mathrm{rps}}\left(\phi_{1}\right)\right| \xi\right\rangle=\left\langle\xi\left|\hat{U}_{\mathrm{rps}}\left(-\phi_{2}\right) \hat{U}_{\mathrm{rps}}\left(\phi_{1}\right)\right| \xi\right\rangle \\
& \quad=\left\langle\xi\left|\hat{U}_{\mathrm{rps}}\left(\phi_{1}-\phi_{2}\right)\right| \xi\right\rangle
\end{aligned}
$$

where the unitarity of the generalized beam splitter has been used. The definition of the relative-phase shift operator has also been used. The scalar product of the right hand side is

$$
\mathrm{e}^{\mathrm{i}\left(\zeta-\zeta^{\prime}\right)}\langle 0 \mid \Psi\rangle\langle\Phi \mid 0\rangle=\mathrm{e}^{\mathrm{i}\left(\zeta-\zeta^{\prime}\right)}\langle 0,0 \mid \xi\rangle\langle\xi \mid 0,0\rangle=\mathrm{e}^{\mathrm{i}\left(\zeta-\zeta^{\prime}\right)}|\langle 0,0 \mid \xi\rangle|^{2},
$$

which follows from the lossless operation (particle number conservation) of the unitary beam splitter. Combining the results of Eqs. (23) and (24) gives a condition for unit visibility

$$
\left|\left\langle\xi\left|\hat{U}_{\mathrm{rps}}(\phi)\right| \xi\right\rangle\right|=|\langle 0,0 \mid \xi\rangle|^{2} .
$$

This is different from the definition of well-defined relative-phase given by Eq. (4). The reason is as follows: If the initial state $|\xi\rangle$ has a vacuum component we see that any state obtained from it by a relative-phase shift also will have a vacuum component since $\hat{U}_{\text {rps }}(\phi)|0,0\rangle=|0,0\rangle$. If the photon counters see no photons (detecting the vacuum) it is impossible to tell different relative-phase shifts apart. However, the vacuum component does not change the visibility.

\subsection{The two-mode coherent state, an example}

Let $|\xi\rangle$ be a two-mode coherent state with equal complex amplitudes in the two modes

$$
|\alpha, \alpha\rangle=\mathrm{e}^{-|\alpha|^{2}} \sum_{n, m=0}^{\infty} \frac{\alpha^{n} \alpha^{m}}{\sqrt{n !} \sqrt{m !}}|n, m\rangle .
$$

Then the relative phase shift gives

$$
\begin{aligned}
& \hat{U}_{\text {rps }}(\phi)|\alpha, \alpha\rangle=\mathrm{e}^{-|\alpha|^{2}} \sum_{n, m=0}^{\infty} \frac{\left(\mathrm{e}^{\mathrm{i} \phi / 2} \alpha\right)^{n}\left(\mathrm{e}^{-\mathrm{i} \phi / 2} \alpha\right)^{m}}{\sqrt{n !} \sqrt{m !}}|n, m\rangle \\
& =\left|\mathrm{e}^{\mathrm{i} \phi / 2} \alpha, \mathrm{e}^{-\mathrm{i} \phi / 2} \alpha\right\rangle .
\end{aligned}
$$

We see that this state does not have a well-defined relative-phase since

$$
\left\langle\alpha, \alpha \mid \mathrm{e}^{\mathrm{i} \phi / 2} \alpha, \mathrm{e}^{-\phi / 2} \alpha\right\rangle=\mathrm{e}^{-2|\alpha|^{2}(1-\cos \phi / 2)},
$$

which is greater than zero for all $\phi$ and $\alpha$, in contrast to what is required by Eq. (4). The probability to find the coherent state in the vacuum state is $|\langle 0,0 \mid \alpha, \alpha\rangle|^{2}=$ $\mathrm{e}^{-2|\alpha|^{2}}$. This means that the condition for unit visibility, Eq. (25), is satisfied for 


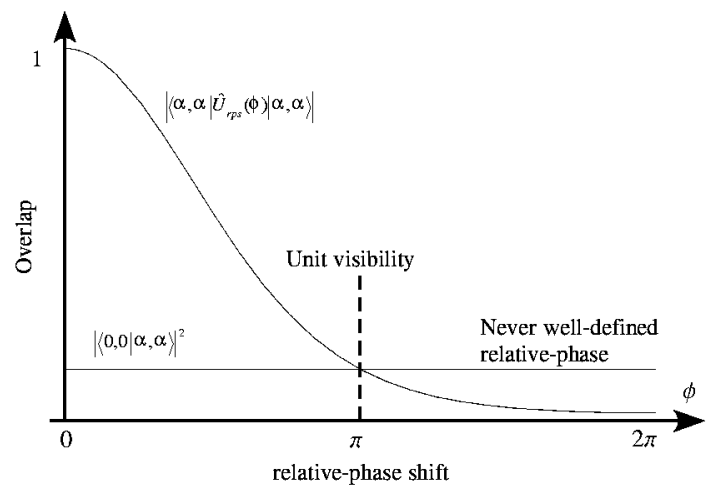

Fig. 3. A plot of $\left\langle\alpha, \alpha\left|\hat{U}_{\mathrm{rps}}(\phi)\right| \alpha, \alpha\right\rangle$. At $\phi=\pi$ the condition for unit visibility is satisfied, but the condition for well-defined relative-phase is never satisfied. Hence, the classical coherent state is an example of a state which may exhibit unit visibility in interferometry but lacks a well-defined relative-phase.

$\phi=\pi$ even if the relative-phase between the two modes is not well-defined. This is probably easiest to understand when the coherent state is viewed as a superposition of binomial states, with a vacuum component. This property is illustrated in Fig. 3 .

\section{Conclusions}

We have illustrated some properties of the relative-phase operator. A state with well-defined relative phase is defined operationally by Eq. (4). To resolve changes in relative-phase well it is argued that relative-phase states [13] are optimal because of their highly entangled nature. Using tailored quantum states in interferometers enhances their resolving power beyond what is possible using classical light. Unfortunately, such states are hard to produce and require very special detection techniques since the commonly used visibility differs from the concept of well-defined relative-phase.

We thank A. Trifonov, S. Inoue, and F. Bretenaker for many fruitful discussions on the topics of this paper. B.H. thanks EOS for the invitation to EOSAM2001 where this work was presented.

\section{References}

[1] A.A. Michelson, Am. J. Sci. 22, 120 (1881).

[2] L. Zehnder, Z.f. Instrkde 11, 275 (1891).

[3] L. Mach, Z.f. Instrkde 12, 89 (1892).

[4] G. Björk, S. Inove, J. Söderholm, Phys. Rev. A 62, 023817 (2000). 
[5] A.A. Michelson, Philos. Mag. 30, 1 (1890).

[6] D. Stoler, B.E.A. Saleh, M.C. Teich, Opt. Acta 32, 345 (1985).

[7] J.M. Radcliffe, J. Phys. A 4, 313 (1971).

[8] Y. Castin, J. Dalibard, Phys. Rev. A 55, 4330 (1997).

[9] K.S. Thorne, Rev. Mod. Phys. 52, 285 (1980).

[10] E. Schrödinger, Proc. Cambridge Philos. Soc. 31, 555 (1936); 32, 446 (1936).

[11] N. Margolus, L.B. Levitin, Physica D 120, 188 (1998).

[12] N. Horsh, A. Mann, J. Phys. A 31, L609 (1998).

[13] A. Luis, L.L. Sánchez-Soto, Phys. Rev. A 48, 4702 (1993).

[14] D. Bouwmeester, J.W. Pan, K. Mattle, M. Eibl, H. Weinfurter, A. Zeilinger, Nature 390, 575 (1997).

[15] H. Weinfurter, M. Zukowski, Phys. Rev. A 64, 010102(R) (2001).

[16] A. Trifonov, T. Tsegaye, G. Björk, J. Söderholm, E. Goobar, M. Atatre, A.V. Sergienko, J. Opt. B 4, 105 (2000).

[17] T. Tsegaye, J. Söderholm, M. Atatre, A. Trifonov, G. Björk, A.V. Sergienko, B.E.A. Saleh, M.C. Teich, Phys. Rev. Lett. 85, 5013 (2000).

[18] P. Usachev, J. Söderholm, G. Björk, A. Trifonov, Opt. Commun. 193, 161 (2001). 